\title{
PERFORMANCE MEASUREMENT OF TRANSACTION-BASED INTERNET APPLICATIONS THROUGH SNMP
}

\author{
Luciano Paschoal Gaspary, Ederson Canterle \\ Programa Interdisciplinar de Pós-Graduação em Computação Aplicada,Universidade do Vale do Rio dos Sinos \\ Av. Unisinos 950 - 93.022-000 - São Leopoldo, Brazil \\ Email:paschoal@exatas.unisinos.br,canterle@terra.com.br
}

\begin{abstract}
Keywords: Transaction-based applications, response time, passive measurement, SNMP.
Abstract: This paper proposes an approach to monitor response time of transaction-based Internet applications and protocols that uses a passive network traffic monitoring technique and stores the resulting statistics in a management information base compatible with the SNMP architecture. The work is within the scope of the Trace platform, which provides support for high-layer protocols, services and networked applications management. The implementation of the proposed approach comprises the extension of the monitoring agent, a key component of the platform, so that it stores information related to response time and generates performance-related reports.
\end{abstract}

\section{INTRODUCTION}

The growing use of computer networks to support applications that require high availability and performance, such as web portals and e-commerce applications, has triggered the investigation of mechanisms that not only assure good performance of the network physical infrastructure, but also let one check the quality of the services and applications running on them. One of the most common metrics to assess the functional state of an application is its performance. Within this context, performance is understood as the response capacity of both the infrastructure and a given application in a networked environment (Sturm, 1999). One of the most important benchmarks to assess the performance of applications is the response time of transactions (protocol interactions).

There are several commercial applications concerned with offering solutions to measure the performance of applications. VitalSuite (Lucent, 2002), Pegasus Network and Application Monitor (NetIQ, 2002), Application Performance Management (Tivoli, 2002), ETE Watch (Candle, 2002), eHealth (Concord, 2002), AppScout (NetScout, 2002) and Spectrum (Aprisma, 2002) are examples of such applications. Among the approaches used to measure response time, five stand out. One of them consists of having software agents installed at end users' stations. These agents monitor user-run transactions, calculate elapsed times and regularly report results to a central station. VitalSuite, ETEWatch, Pegasus Application Monitor, Application Performance Management and Spectrum are examples of management applications that use agents at users' stations. A variation of this approach is implemented by eHealth and Spectrum applications, consisting of agents at server stations to monitor the behavior of applications (for instance, by permanently watching log files). The third approach follows the execution of artificial transactions triggered from stations located at locations where it is important to measure the performance users perceive. VitalSuite, Pegasus Network Monitor, Application Performance Management and Spectrum offer this kind of functionality. The fourth and most powerful approach, used by the Tivoli platform, is based on instrumenting the application to be monitored on client and/or server ends. Finally, the fifth approach obtains information by passively monitoring network traffic. AppScout and VitalSuite implement this technique.

The first four approaches are invasive. The employment of agents at end-user or server stations implies the use of resources and loss of performance at the stations where they are installed. The use of synthetic transactions to assess application performance has the disadvantage of using additional network resources. Instrumenting software is useful to monitor applications built within the organization, but it cannot be used to monitor proprietary protocols and ap- 
plications. Also, it takes considerable investments to train personnel in using programming APIs (Application Programming Interfaces).

Besides being invasive, most current approaches of the available management applications are proprietary, which induces organizations to acquire management software from a single manufacturer even if it does not satisfactorily meet most needs. It thus becomes a complex task to integrate the information generated by such applications to the management platforms organizations already use, which impairs an integrated view of the operation of both the network infrastructure and applications.

This paper presents an approach to monitor response time of transaction-based Internet applications and protocols that (a) uses a passive network traffic monitoring technique and (b) stores resulting statistics in an SNMP-compatible management information base (which allows statistics to be obtained from any management application that supports SNMP). The work is within the scope of the Trace platform, which offers support to manage high-layer protocols, services and applications (Gaspary et al., 2002). The implementation of the proposed approach covers the extension of the monitoring agent, a key component of the platform, so that it stores response time-related information and generates performance reports, beside monitoring the number of trace occurrences. The specification of protocol interactions whose response time is to be measured is performed in PTSL (Protocol Trace Specification Language), as proposed by (Gaspary et al., 2001). The network manager uses these specifications to remotely configure the monitoring agents through the Script MIB (Levi and Schönwälder, 2001). In order to configure performance measures to be carried out and to retrieve results, the manager interacts with the agent, also via SNMP, through a subset of the Application Performance Measurement MIB (Waldbusser, 2002).

The main contributions of our work are twofold. First, we have developed a solution to measure protocol and application interactions response time that, at the same time, uses a passive technique and provides results via SNMP (allowing its integration to network management systems already in use). Second, we propose a more flexible mechanism to specify, on the fly, the transactions whose response time are supposed to be measured (in substitution to the mechanism provided by APM).

This paper is structured as follows: section 2 summarizes how to use PTSL to represent the protocol interactions to be monitored. Next, section 3 presents the subset of the Application Performance Measurement MIB used. The paper follows with a detailed presentation of the architecture of the monitoring agent in section 4 . Section 5 mentions some examples of the use of the agent and section 6 con- cludes with some final considerations and prospects for future research.

\section{REPRESENTATION OF INTERACTIONS TO BE MONITORED}

This section summarizes the language used to represent protocol traces called PTSL (Protocol Trace Specification Language), originally described in (Gaspary et al., 2001). Through this language, the network manager must specify the protocol interactions (transactions) whose response times are to be measured. The language is based on the concept of finite state machines, and it comprises graphical (Graphical PTSL) and textual (Textual PTSL) notations. The notations are not equivalent. The textual notation allows the complete representation of a trace, including the specification of the state machine and the events that trigger transitions. The graphical notation, in turn, is equivalent to a subset of the other, and offers the possibilities of depicting the state machine and only labeling the events that enable transitions.

Figures 1 and 2 illustrate some protocol traces described using the language's graphical notation. The trace shown in figure 1 allows monitoring HTTP requests that will be successfully returned, and it can be used to gather response times of successful accesses to a web server. This kind of information can be rather useful for companies that host web sites, portals, and e-commerce applications (Application Service Providers) because it allows the identification of regions from where user-perceived response time is too long. The response time of other interactions of the HTTP protocol can be monitored similarly. For instance, the time elapsed between the submission of a given form via browser and the corresponding return can be measured with the specification of a trace where the request GET of figure 1 is replaced with something like POST/path/script.cgi $\operatorname{HTTP} / 1.1$.

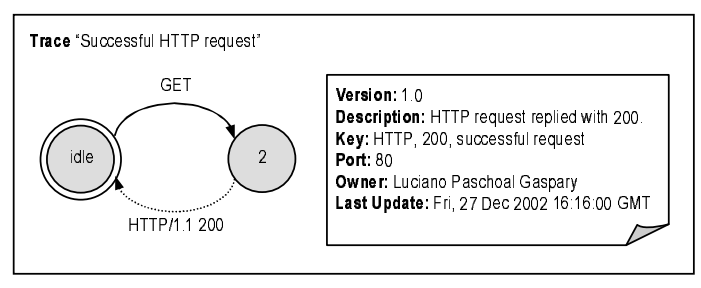

Figure 1: Trace to monitor successful HTTP requests

As can be seen in figure 1, states in the graphical notation are represented by circles. From the initial 
idle state, $n$ other states can be created, as long as they can be reached through some transition. The final state is identified by two concentric circles. Transitions are represented by single-pointed arrows. A continuous arrow means the transition is triggered by the client station, whereas a broken arrow means that the transition is triggered by a server station event. The text associated to a transition merely labels the event that triggers the transition; its specification can be made through the textual notation.

Figure 2 illustrates other examples. (a) and (b) present traces that model a user's authentication at a POP3 server. In (a) the username is informed and in (b) so is the password. These traces can be used to store the response time of each stage of the authentication process. If the manager is interested in storing a single response time comprising the entire authentication process, the two traces can be combined. In such case, the resulting trace would have four states: idle $\rightarrow 2 \rightarrow 3 \rightarrow 4 \rightarrow$ idle. Other significant protocol interactions can be modeled by replacing transition USER in (a) with LIST, RETR or DELE, to mention just a few. The trace shown in (c) allows monitoring the response time of requests to a DNS server. PTSL also allows modeling protocol traces that do not belong to the application layer. For instance, (d) specifies the three steps to open a TCP connection (threeway handshaking). This trace can be used to measure the time spent to start a TCP connection between any two end-points.
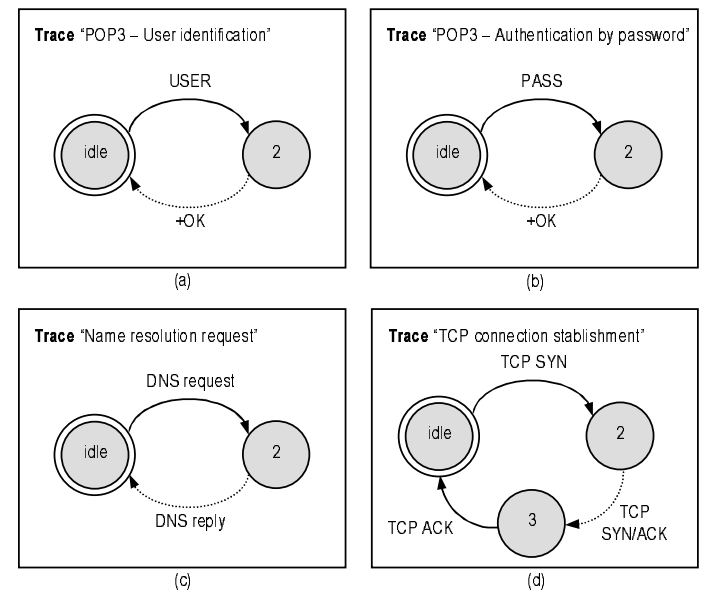

Figure 2: Graphical representation of protocol traces

Figure 3 presents the textual specification of the trace illustrated in figure 1 . Every specification written in Textual PTSL begins with the word Trace and ends with EndTrace (lines 1 and 37). Catalog and version control information appear right after the keyword Trace (lines 2 to 7). After that, the specification is divided into three sections: Message Section (lines 9 to 22), Groupsection (not used in this example) and Statessection (lines 24 to 35). MessagesSection and GroupSection define the messages that trigger the evolution of the trace. Statessection defines the state machine that represents the trace.

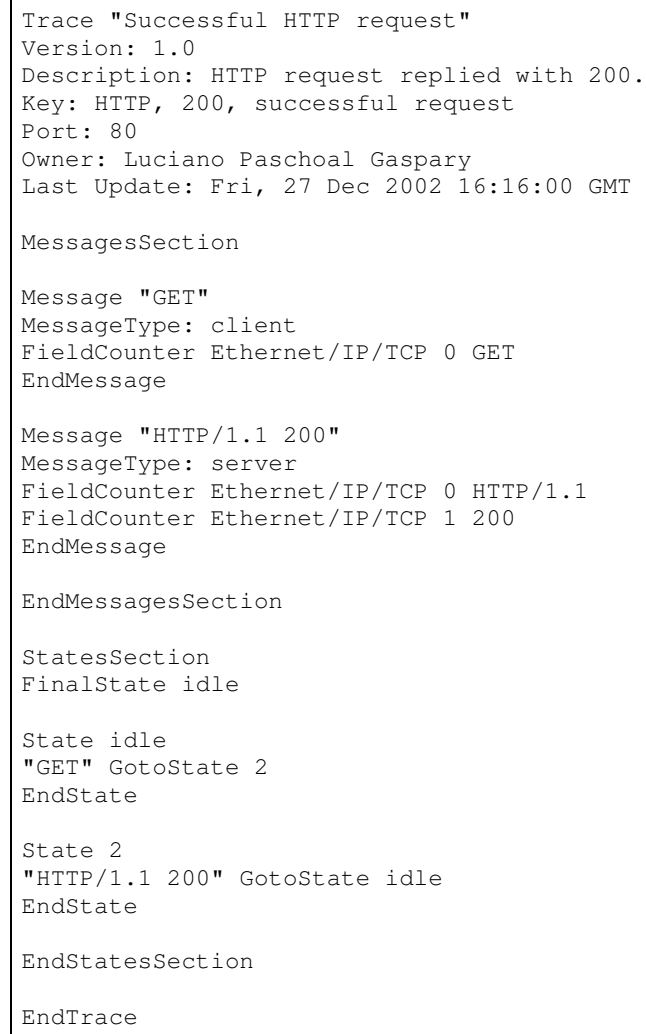

Figure 3: Textual representation of a trace

The event that triggers the evolution of the state machine is the verification of a packet on the network that contains fields with values equivalent to those specified in a message (Message). The way to specify the fields to be analyzed depends on the type of protocol to be monitored. In the case of characterbased protocols, which have variable-length fields separated by blank spaces (e.g. HTTP and POP), a field is identified by its position in the message (FieldCounter strategy). In HTTP/1.1 200, for instance, $\operatorname{HTTP} / 1.1$ is at position 0 and 200 at position 1 of the message. On the other hand, field identification in binary protocols, which feature fixedlength fields (e.g. TCP and DNS), is determined by a bit offset from the beginning of the protocol header to the beginning of the desired field; besides the initial position of the field, it is also necessary to inform the number of bits the field uses (Bit Counter strat- 
Table 1: Sample performance report generated by the APM MIB

\begin{tabular}{|c|ccc|l}
\cline { 2 - 4 } \multicolumn{1}{c|}{} & Req. HTTP & Req. HTTP & Req. HTTP & Trace \\
& 172.16 .108 .1 & 172.16 .108 .32 & 172.16 .108 .1 & Client \\
\hline TransactionCount & 172.16 .108 .2 & 172.16 .108 .2 & 200.248 .252 .1 & Server \\
SuccessfulTransactions & 125 & 500 & 313 & \\
ResponsivenessMean & 100 & 447 & 200 & \\
ResponsivenessMin & 4.50 & 3.25 & 5.25 & \\
ResponsivenessMax & 0.75 & 0.85 & 0.99 & Ranges of \\
ResponsivenessB1 & 5.85 & 5.00 & 5.90 & performance \\
ResponsivenessB2 & 5 & 27 & 10 & $0 \leq$ t.r. $<1$ \\
ResponsivenessB3 & 10 & 65 & 17 & $1 \leq$ t.r. $<2$ \\
ResponsivenessB4 & 28 & 152 & 50 & $2 \leq$ t.r. $<3$ \\
ResponsivenessB5 & 20 & 142 & 30 & $3 \leq$ t.r. $<4$ \\
ResponsivenessB6 & 25 & 37 & 40 & $4 \leq$ t.r. $<5$ \\
& 12 & 24 & 53 & $5 \leq$ t.r. $<6$ \\
\end{tabular}

egy).

The trace shown in figure 1 is for a character-based protocol. Specification of message GET is illustrated in figure 3 (lines 11 to 14 ). Line 12 defines the message as being of the client type, which means that the state transition associated to the message will be triggered by the client station. Line 13 specifies the only field to be analyzed. The information required to identify it is: location strategy (FieldCounter), protocol encapsulation (Ethernet/IP/TCP), field position (0), expected value (GET) and an optional field description. Message HTTP/1.1 200 (lines 16 to 20 ) is specified in a similar way. More information on PTSL and more examples of traces can be found at (Gaspary et al., 2001).

\section{THE APPLICATION PERFORMANCE MANAGEMENT MIB}

The APM (Application Performance Management) MIB, proposed by (Waldbusser, 2002), defines objects that provide performance statistics (e.g. response time) that users perceive in the applications they use. The methods employed to build these statistics are not specified by the MIB, so that each manufacturer can choose those considered more convenient. Originally designed to furnish information on transaction, throughput and streaming-oriented application performance, the MIB has been simplified by our research group to offer support to the first type only (transaction-oriented), which are the target applications of the Trace platform (Gaspary et al., 2002). Henceforth we are going to call it short APM.

\subsection{Performance Reports}

The traces observed by an APM agent can be aggregated in several ways to generate statistical performance reports. The granularity of these reports and how often they ought to be generated may be specified by the network manager. The statistics the MIB offers after aggregating a set of traces are as follows: (a) TransactionCount: number of trace occurrences in the interval; (b) SuccessfulTransactions: number of successfully completed traces; (c) ResponsivenessMean: arithmetic mean of all successfully completed aggregated traces; (d) ResponsivenessMin: minimum response time observed among all successfully completed aggregated traces; (e) ResponsivenessMax: maximum response time observed among all successfully completed aggregated traces; (f) $R e$ sponsivenessBx: number of successfully completed aggregated traces whose performance matches one of six specified ranges. Because the performance of each application varies a lot, the values of these ranges can be specified separately for each monitored trace.

The APM MIB supports four different types of aggregation: (a) aggregation of flows: for this kind of aggregation, a record is created for each different combination of trace, client and server the agent observes; (b) aggregation of clients: a record is created for each different combination of trace and client; (c) aggregation of servers: a record is created for each different combination of trace and server; (d) aggregation of applications: a record is created for each trace observed.

Table 1 illustrates a hypothetical example of a report with performance information related to a set of traces Successful HTTP request, aggregated by flow and monitored over a given time interval. It can be seen that 125 HTTP traces occurred between stations 172.16.108.1 and 172.16.108.2. Out of these 125 traces, 100 were successfully completed. The mean 


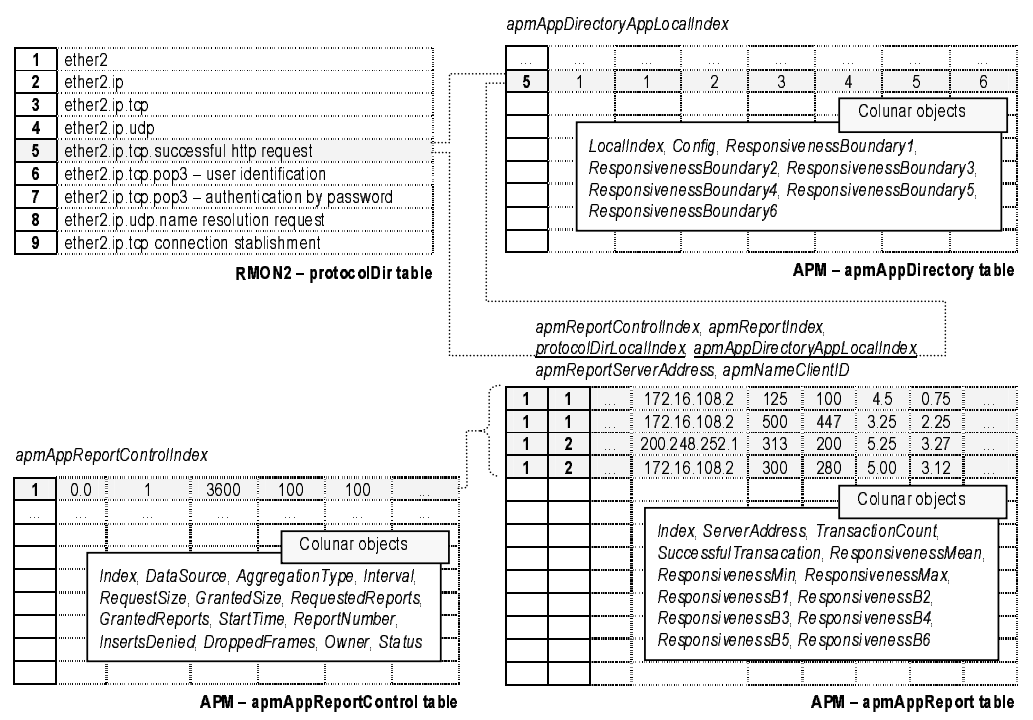

Figure 4: Tables of the short APM MIB

response time of successful traces was 4.50 seconds. The fastest trace took 0.75 seconds to complete and the slowest took 5.85 seconds. It can also be seen that most successful traces had response time from 2.00 to 5.00 seconds.

\subsection{MIB Structure}

In order to offer the statistics mentioned, the short APM MIB comprises two groups: apmAppDirectory and apmReport. The first one is used to perform configurations of the monitored applications, including the six performance ranges (table apmAppDirectory). The second group, in turn, controls the creation and retrieval of performance reports of these applications. In order to do so, it has two tables: apmAppReport Control and apmAppReport. Figure 4 shows these tables.

Table apmAppDirectory has one entry for each trace to be monitored ${ }^{1}$. This table allows one to configure, for each trace, whether the APM agent should collect performance statistics for the trace and the six performance ranges. Figure 4 illustrates the entry related to the Successful HTTP request trace in table apmAppDirectory. An agent configured this way is able to measure the response time of such trace (apmAppDirectoryConfig=1) and its performance ranges are $0-1,1-2,2-3,3-4,4-5$ and 5-6 seconds.

Table apmAppReportControl lets one configure the parameters to generate performance reports

\footnotetext{
${ }^{1}$ Traces to be monitored are defined in table protocolDir of the RMON2 MIB (Gaspary et al., 2001).
}

on the observed traces. Each entry in this table corresponds to a kind of report to be generated and has the following information: monitored interface (apmAppReportControlDataSource), level of aggregation (apmAppReportControlAggregationType), monitoring

interval

(apmAppReportControl Interval),

number of records admitted in report (apmAppReportControlGrantedSize),

number of storable reports (apmAppReportControlGrantedReports), and others. In figure 4 , the requested report will be generated from monitoring the standard interface (0.0), the traces will be aggregated by flows (1), the monitoring interval will be 3600 seconds and the report will not admit more than 100 records. The maximum number of storable reports is 100 .

Finally, the task of table apmAppReport is to store the records generated for the configured reports. The information available in this table was specified in the previous sub-section. As figure 4 shows, the entries available in table apmAppReport belong to two consecutive reports generated from the configuration of table apmAppReportControl. In order to retrieve the number of Successful HTTP request traces successfully completed (apmAppReportTransactionCount) between stations 172.16.108.1 and 172.16.108.2 in the first report, the OID to be used is apmReportTransactionCount.1.1.5.5. 172.16 .108 .1 .172 .16 .108 .2 . 


\section{AGENT'S ARCHITECTURE}

The SNMP agent that assesses performance is input with protocol traces specified with PTSL language. The configuration of the traces to be monitored at a given time is performed by the manager, who communicates with the agent through the Script MIB. Once programmed, it starts monitoring trace occurrence. In order to configure performance measurements to be carried out, the manager interacts with the agent, via SNMP as well, through the subset of the Application Performance Measurement MIB presented in section 3 . The resulting reports can be retrieved from any SNMP-supporting application through regular polls to the APM agent. Figure 5 illustrates the flow of information between manager and agent.

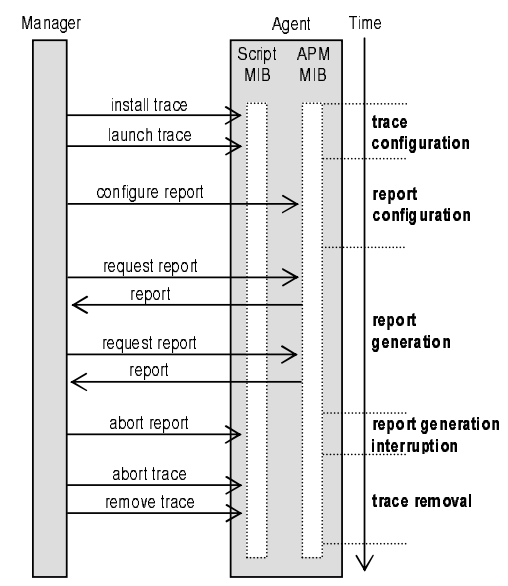

Figure 5: Flow of information between manager and APM agent

The monitoring agent runs on Linux stations and was implemented using the $\mathrm{C}$ language, the POSIX thread library (Pthreads, 1995) and the NET-SNMP framework (NET-SNMP, 2002). A more detailed view of the agent's architecture is shown in figure 6. The PTSL manager thread is responsible for integrating the Script MIB and the PTSL core. It updates the structures used by the PTSL core every time a new trace is programmed to be monitored or an existing trace is deleted (as no longer necessary). Three other threads - queue, PTSL engine and APM - operate in a producer/consumer fashion. The first captures all the packets arriving at the network interface card using the libpcap library and adds them to a circular queue. Although this library supports filter specification with the BPF (BSD Packet Filter) notation (Mccanne and Jacobson, 1993), the agent is not using this feature. The second thread processes each packet in the queue without deleting it, aiming to verify whether it has the expected features to allow one or more traces to evolve in the state machine. If so, the packet is marked. The APM thread, in turn, removes each packet from the queue and, according to the marks and configured reports, updates a memorybased data structure. As soon as the monitoring interval of a report is over, the information in the memory is processed and the results are stored in a database (mySQL). This database is queried by an extension to the NET-SNMP agent that implements the short APM MIB.

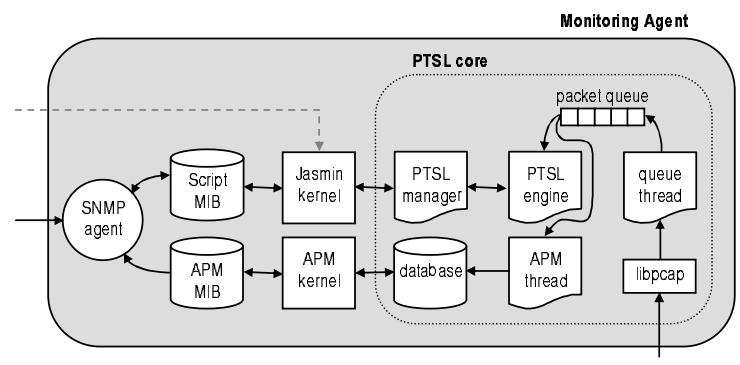

Figure 6: Agent's internal architecture

Regarding security, the agent supports all capabilities of the SNMPv3 protocol, including models USM (User-based Security Model) (Blumenthal and Wijnen, 1999) and VACM (View-based Access Control Model) (Wijnen et al., 1999). Their use prevents unauthorized people from reconfiguring the agent.

\section{EXAMPLES OF USE FOR THE AGENT}

Monitoring the response time of protocol, service and networked application interactions is a rather relevant task when these programs are used to support critical operations, which require high availability and performance. Several scenarios can be used to illustrate the need to warrant such requirements. Two are shown below: application service providers and corporate networks.

\subsection{Application Service Providers}

Application service providers must warrant 24-houra-day, 7-day-a-week access to users/clients. Given the extreme competition between companies that offer similar hosting services, high availability ought to be accompanied by low response time. Companies that hire hosting services to make sure their users/clients have efficient access to sites, portals and so on (within hired specifications of quality) depend on applications that monitor actual accesses. In this scenario, the agent we present can be used by the provider and/or 


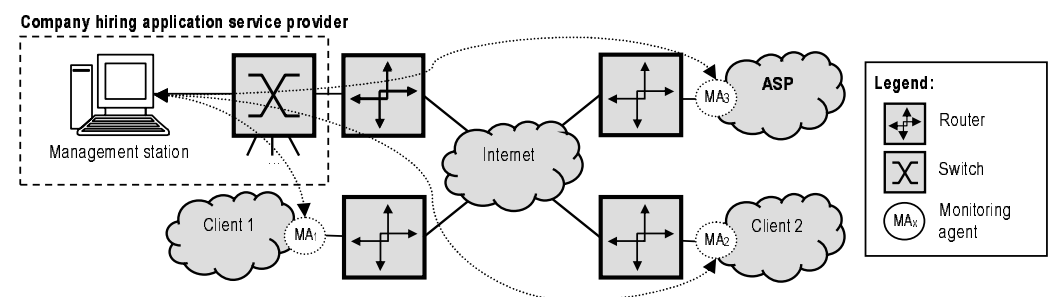

Figure 7: Monitoring a hosting environment

the internal network of some users/clients. Regardless of the location of the agent, the party hiring the hosting service can remotely configure the traces and reports desired, and retrieve results likewise with some management tool that supports SNMP (see figure 7).

\subsection{Corporate Networks}

The corporate network environment is another recommended scenario for monitoring the response time of protocol, service and networked application interactions. Within that environment, not only sites and portals (e.g. intranet), but also essential services such as DNS and POP should have their response times measured, as well as applications of the organization.

Figure 8 illustrates a real management scenario. The router, through a serial interface, is the link to the Internet. Besides the serial interface, the router features two Ethernet interfaces. The first one is connected to a hub, which is linked to two stations: one hosts DNS and the other hosts POP. The second interface of the router is linked to a station with two network interfaces. It runs a firewall and therefore represents the borderline between the internal and external networks. Linked to the other interface of the firewall (internal interface) is a hub. The web server responsible for the Intranet of the organization and a switch that segments the internal network into several subnetworks are linked to it. The other equipment (blank in the figure) are the management station and two stations dedicated to the monitoring task.

In order to monitor response time of accesses to the Intranet server, agent $\mathrm{MA}_{1}$ must be configured with traces such as Successful HTTP request, illustrated in figure 1. Likewise, agent $\mathrm{MA}_{2}$ must be programmed with traces such as POP3 - User identification, POP3 - Authentication by password and Name resolution request to monitor the response time of accesses to POP and DNS servers. After traces are configured, interaction with the agents through the APM MIB is needed to request the generation of performance reports (creation of one or more entries in table apmAppReportControl, presented in subsection 3.2). Results can be later obtained by querying table apmAppReport.

\section{CONCLUSIONS AND FUTURE WORK}

This paper has presented an agent to monitor response time of protocol interactions that, unlike the available solutions, combines together two essential features: uses the technique of passive monitoring of network traffic and stores resulting statistics in an SNMP-compatible management information base. Sections 2 and 3 presented PTSL, a language used to specify the protocol interactions to be monitored, and then the short APM MIB, interface of the manager with the agent-generated performance statistics. The internal architecture of the agent and some samples of its usage were explained in sections 4 and 5 .

Regarding the APM MIB originally proposed in (Waldbusser, 2002), it should be stressed that it has been under standardization for at least four years and presentes several divergent points. The original MIB is lengthy and hard to implement, much due to the support to many kinds of applications (transaction, throughput and streaming-oriented). It is also limited regarding monitorable transactions. A documentation that is also under standardization (Bierman et al., 2002) defines well known and spread protocol transactions that APM agents should support. However, the most important applications for organizations are proprietary and usually developed within the companies themselves. These applications will never be registered as standard and APM agent manufacturers will hardly be able to include support to them in their products. These limitations led to MIB adaptations, simplifying it to support a specific class of applications - transaction-oriented - and replacing the identification mechanism of transactions to be monitored with a much more flexible one, which the network manager can configure with PTSL.

The expression power of PTSL should be highlighted. This language allows the specification of protocol interactions at network, transport and application layers. Also, unlike existing solutions, it allows modeling interactions of both conventional protocols and organization's applications. In order to specify transactions related to encrypted protocols (e.g. Secure Electronic Transaction), traces are described 


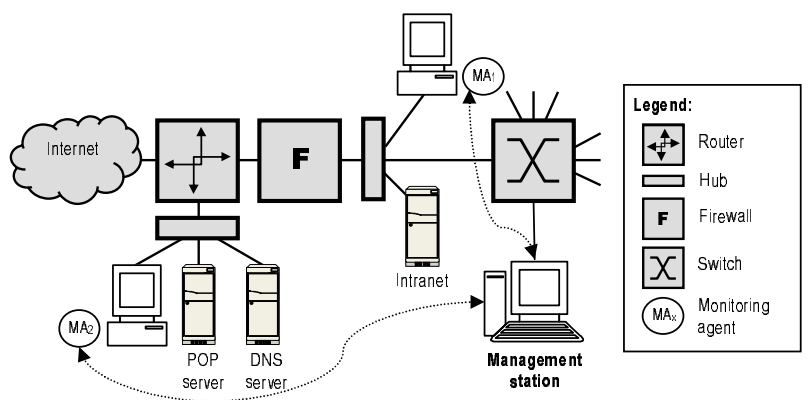

Figure 8: Monitoring a corporate environment

similarly. However, the agent will have to be remodeled so as to intercept and decode observed messages.

Although the approach based on the passive monitoring of network traffic is the least invasive, it has a greater computational cost. Measurements performed by our research group allowed us to detect that the low packet-processing capacity of the agent is due to the broad use of a data base (mySQL) to consolidate performance-related statistics. From that observation, the first implementation of the agent has been redesigned to substitute database for memory data structures. Among structures considered, hash tables were the most appropriate because they allow fast input and update of elements. At present, there is an ongoing project to gradually implement the designed changes.

\section{REFERENCES}

Aprisma (2002). Aprisma Technologies Homepage. http://www.aprisma.com/.

Bierman, A., Bucci, C., Dietz, R., and Warth, A. (2002). Remote Network Monitoring MIB Protocol Identifier Reference Extensions. RFC 3395.

Blumenthal, U. and Wijnen, B. (1999). User-based Security Model (USM) for version 3 of the Simple Network Management Protocol (SNMPv3). RFC 2574.

Candle (2002). Candle Corporation Homepage. http://www.candle.com.

Concord (2002). Concord Communications Inc. Homepage. http://www.concord.com.

Gaspary, L., Balbinot, L. F., Storch, R., Wendt, F., and Tarouco, L. (2001). Uma arquitetura para gerenciamento distribuído e flexível de protocolos de alto nível e serviços de rede. In IXX Simpósio Brasileiro de Redes de Computadores.

Gaspary, L., Meneghetti, E., Wendt, F., Braga, L., Storch, R., and Tarouco, L. (2002). Trace: An open platform for high-layer protocolos, services and networked applications management. In Proc. of the
VIII IEEE/IFIP Network Operations and Management Symposium, pages 871-873.

Levi, D. and Schönwälder, J. (2001). Definitions of Managed Objects for the Delegation of Management Scripts. RFC 3165.

Lucent (2002). Lucent Technologies Homepage. http://www.lucent.com/.

Mccanne, S. and Jacobson, V. (1993). The bsd packet filter: A new architecture for user-level packet capture. In Proc. of the USENIX CONFERENCE, pages 259-269.

NET-SNMP (2002). NET-SNMP Project Homepage. http://net-snmp.sourceforge.net/.

NetIQ (2002). NetIQ Corporation Homepage. http://www.netiq.com/.

NetScout (2002). NetScout Systems, Inc. Homepage. http://www.netscout.com/.

Pthreads (1995). Pthreads: POSIX threads standard. IEEE Std 1003.1c-1995.

Sturm, R. (1999). Foundations of Application Management. John Wiley \& Sons.

Tivoli (2002). Tivoli Systems, Inc. Homepage. http://www.tivoli.com.

Waldbusser, S. (2002). Application Performance Measurement MIB. Internet Draft.

Wijnen, B., Presuhn, R., , and McCloghrie, K. (1999). Viewbased Access Control Model (VACM) for the Simple Network Management Protocol (SNMP). RFC 2575. 